\title{
Cell-based simulations of biased epithelial lung growth
}

\section{Journal Article}

Author(s):

Stopka, Anna; Meer, Marco (D); lber, Dagmar (D)

Publication date:

2019-12-12

Permanent link:

https://doi.org/10.3929/ethz-b-000376937

\section{Rights / license:}

Creative Commons Attribution-NonCommercial-NoDerivs 3.0 Unported

Originally published in:

Physical Biology 17(1), https://doi.org/10.1088/1478-3975/ab5613

\section{Funding acknowledgement:}

170930 - A 3D Cell-Based Simulation Framework for Morphogenetic Problems (SNF) 


\section{Cell-based simulations of biased epithelial lung growth}

To cite this article before publication: Anna Stopka et al 2019 Phys. Biol. in press https://doi.org/10.1088/1478-3975/ab5613

\section{Manuscript version: Accepted Manuscript}

Accepted Manuscript is "the version of the article accepted for publication including all changes made as a result of the peer review process, and which may also include the addition to the article by IOP Publishing of a header, an article ID, a cover sheet and/or an 'Accepted Manuscript' watermark, but excluding any other editing, typesetting or other changes made by IOP Publishing and/or its licensors"

This Accepted Manuscript is @ 2019 IOP Publishing Ltd.

During the embargo period (the 12 month period from the publication of the Version of Record of this article), the Accepted Manuscript is fully protected by copyright and cannot be reused or reposted elsewhere.

As the Version of Record of this article is going to be / has been published on a subscription basis, this Accepted Manuscript is available for reuse under a CC BY-NC-ND 3.0 licence after the 12 month embargo period.

After the embargo period, everyone is permitted to use copy and redistribute this article for non-commercial purposes only, provided that they adhere to all the terms of the licence https://creativecommons.org/licences/by-nc-nd/3.0

Although reasonable endeavours have been taken to obtain all necessary permissions from third parties to include their copyrighted content within this article, their full citation and copyright line may not be present in this Accepted Manuscript version. Before using any content from this article, please refer to the Version of Record on IOPscience once published for full citation and copyright details, as permissions will likely be required. All third party content is fully copyright protected, unless specifically stated otherwise in the figure caption in the Version of Record.

View the article online for updates and enhancements. 


\title{
Cell-based Simulations of Biased Epithelial Lung Growth
}

\author{
Anna Stopka ${ }^{1,2}$, Marco Kokic ${ }^{1,2} \&$ Dagmar Iber ${ }^{1,2}$
}

${ }^{1}$ Department of Biosystems Science and Engineering, ETH Zürich, Switzerland

${ }^{2}$ Swiss Institute of Bioinformatics, Switzerland

E-mail: dagmar.iber@bsse.ethz.ch

\begin{abstract}
During morphogenesis, epithelial tubes elongate. in the case of the mammalian lung, biased elongation has been linked to a bias in cell shape and cell division, but it has remained unclear whether a bias in cell shape along the axis of outgrowth is sufficient for biased outgrowth and how it arises. Here, we use our 2D cell-based tissue simulation software LBIBCell to investigate the conditions for biased epithelial outgrowth. We show that the observed bias in cell shape and cell division can result in the observed bias in outgrowth only in the case of strong cortical tension, and comparison to biological data suggests that the cortical tension in epithelia is likely sufficient. We explore mechanisms that may result in the observed bias in cell division and cell shapes. To this end, we test the possibility that the surrounding tissue or extracellular matrix acts as a mechanical constraint that biases growth in the longitudinal direction. While external compressive forces can result in the observed bias in outgrowth, we find that they do not result in the observed bias in cell shapes. We conclude that other mechanisms must exist that generate the bias in lung epithelial outgrowth.




\section{1. Introduction}

2 Epithelial tubes are an essential component of many organs, and their development has been studied in many different model organisms. In Drosophila, where overall growth is limited by the exoskeleton, tube elongation is mostly associated with changes in cell shape, and cell position, which can also include the recruitment of surrounding cells [1]. For example, in the Drosophila salivary/gland and trachea, tubes elongate by cell shape changes in combination with cell migration. In the Drosophila tracheal system, dynamic filopodia and lamellipodia have been observed in response to Fgf signaling, which would support directed cell migration [2]. Mammalian organs grow extensively during development, and it is largely unknown how the outgrowth can become biased [1]. In this article, we focus on the biased elongation of epithelial tubes during mouse lung development.

The developing mouse lung offers examples of both isotropic and anisotropic tube expansion. Thus, the mouse embryonic tracheal epithelium expands isotropically, i.e. the circumference widens to the same extent as the tube elongates [3], whereas the mouse embryonic lung bronchi show biased outgrowth, i.e. the epithelial tube lengthens more than it widens [4, 5]. The biased outgrowth of embryonic mouse lung bronchi has been related to a bias in the orientation of the mitotic spindles of the dividing cells [4]. Similarly, in the mouse kidney, a bias in the orientation of the mitotic spindles of dividing cells is observed during renal tube elongation [6].

But how would the bias in cell division be achieved in the first place? The planar cell polarity (PCP) pathway plays an important role in regulating the mitotic spindle angle distribution [7, 8]. However, while the PCP pathway supports elongating outgrowth in the kidney [6], similar effects are not observed in the embryonic lung [4], and independent of whether or not the PCP pathway is involved, it does not address the question of how the direction of outgrowth is defined in the first place. According to Hertwig's rule, cells divide through their mass point, and perpendicular to their longest axis $[9,10,11]$. Indeed, the bias in cell division is accompanied by a bias in cell shape [5]. Accordingly, we need to understand how the bias in cell shape arises in the direction of elongating outgrowth.

In principle, such a bias could originate from a polarity along the tube, from a pulling force at the tip, or from a mechanical constraint that limits circumferential expansion [12]. The most obvious polarity cue is provided by fibroblast growth factor (Fgf), a well-known chemoattractant for lung buds [13]. However, while lung buds grow towards a source of Fgf in culture, there is no evidence that Fgf signaling gradients exist along outgrowing buds, and bud outgrowth is observed 32 when the epithelium is exposed to uniform Fgf concentrations [14]. In fact, computational modeling 33 suggests that a ligand-receptor based Turing mechanisms concentrates Fgf signaling in spots where 34 new branches grow out $[15,16]$, and thus also contradicts the formation of a gradient along the 35 epithelial tube. Alternatively, Fgf signaling could mediate elongating outgrowth by generating a 36 pulling force at the tip. In fact, Fgf2 signaling has been shown to enhance motility and persistence 37 of epithelial cells at the tip of epithelial tubes in the mammary gland [17]. A pulling force would act 38 on the cells behind the tip via the cell-cell adhesion bonds, and could thus generate a bias in cell 39 shape along the entire tube. in the case of pulling forces at the tip, actin-rich protrusions would be ${ }_{40}$ expected at the tip. However, in the mammary gland, leading cell extensions or specialized actin-rich 
protrusions, such as filopodia and lamellipodia, are not observed at the front of advancing mammary ducts [18]. As an alternative to cell migration, biased outgrowth in the mammary gland has been linked to a mechanical constraint posed by the myoepithelium [19]. Such a mechanical constraint in the circumferential direction would result in growth-dependent uniform expansion in longitudinal direction, and would thus explain the measured linear gradient in cell speeds that is observed along the elongating mammary gland tube [17]. The lung mesenchyme could, in principle, play a similar role in the embryonic lung though smooth muscles mature only at later stages and are not as tightly associated as in a myoepithelium [20]. In the developing kidney, however, smooth muscles are largely absent, and branches elongate also in the absence of mesenchyme [21]. Finally, in plants, hoop stress has been suggested to induce an anisotropy in the cell walls [22], such that growth is limited in circumferential direction. While the cytoplasmic hydrostatic pressure and extracellular matrix (ECM) stiffness are orders of magnitude smaller than in plants, a bias in ECM stiffness could, in principle, limit expansion in circumferential direction also in mammalian organs.

An additional challenge in explaining epithelial outgrowth is posed by the visco-elastic nature of tissue. While cortical tension together with cell-cell adhesion interactions result in an elastic tissue behavior, cellular remodeling, cell migration, cell shape changes and rearrangements [23, 24] result in a fluid-like behavior [25, 26]. Whether the observed bias in cell division is sufficient to explain the bias in outgrowth will depend on the fluidity of the tissue, and on potential external forces that may bias the growth process. In this context, it is interesting to note that the bias in cell division and in epithelial outgrowth is lost in embryonic lungs that express a constitutive active form of KRas $\left(\right.$ Shh $^{\text {cre/+}}$; KRas $\left.{ }^{L S L /+}\right)$ [4], which activates Erk, a target of Fgf signaling [27]. KRas signaling in airway epithelial cells has previously been linked to changes in cell shape and motility by affecting cortical actin [28]. Expression of a constitutive active form of KRas (KRas $\left.{ }^{G 12 D}\right)$ in pancreatic ducts results in a redistribution of phosphorylated myosin light chain 2 (pMLC2) inside the cell, so that its concentration declines on the apical side and increases on the basal side [29]. Constitutive active KRas may thus reduce cortical tension on the apical side of the epithelium, where all cell divisions occur [30].

We sought to use cell-based tissue simulations to explore whether the observed bias in cell division, is, in principle, sufficient to drive the observed bias in outgrowth when taking the viscoelastic tissue properties into account, and whether constricting forces from the surrounding tissue and/or ECM can, in principle, explain the observed bias in cell division, cell shape, and outgrowth. In the cases where tissue behavior emerges from processes at the single cell level, cell-based simulations can be helpful to identify the underlying mechanisms [31]. A wide range of cell-based tissue simulation frameworks have been developed [32]. We chose to use our previously developed 2D cell-based simulation environment LBIBCell to simulate visco-elastic tissue dynamics [33], but we note that vertex models [34] can be expected to yield similar results, even if they have a lower geometric resolution and do not explicitly represent cell-cell adhesion. LBIBCell models the fluid-structure interaction in a biological tissue by combining the Lattice Boltzmann (LB) [35] and Immersed Boundary (IB) [36] methods. Cells are represented as finely resolved polygons according to the IB method. The cell boundaries are elastic and cells interact with their neighbors via spring-like cell-cell junetions. The cytoplasm inside the cells is a fluid, and cells are immersed in a thin layer of 
1 fluid, the interstitial fluid, that provides nutrients to the cells. The fluid behavior inside and outside 2 of the cells is described by the LB equations. Cell-based simulations are particularly useful when 3 quantitative data is available to inform cell-based rather than continuous models $[32,31]$. in the 4 case of epithelia, quantifications of the apical polygonal lattice are available for a wide range of 5 epithelia from Drosophila and mouse $[4,37,38,5,39]$. These can be used toadjust the mechanical 6 parameters in the model $[39,40]$. The additional biological parameters, i.e. the growth rate, the rate 7 of apoptosis and cell division, and the orientation of the cell division axis have all been measured in 8 embryonic mouse lungs. Using LBIBCell, we find that the bias in the cell division axis is sufficient to 9 explain the observed bias in outgrowth only for high cortical tension. A mechanical constraint from 10 the surrounding tissue, as has been proposed for the mammary gland [19], can give rise to biased 11 outgrowth, but fails to explain the observed bias in cell shape and thus cell division orientation. We 12 conclude that the bias in embryonic lung outgrowth can arise from the observed bias in cell division, 13 but the bias in cell shape cannot arise from mechanical constraints imposed by the surrounding 14 tissue. Other mechanisms must thus drive biased epithelial outgrowth of mouse lung tubes.

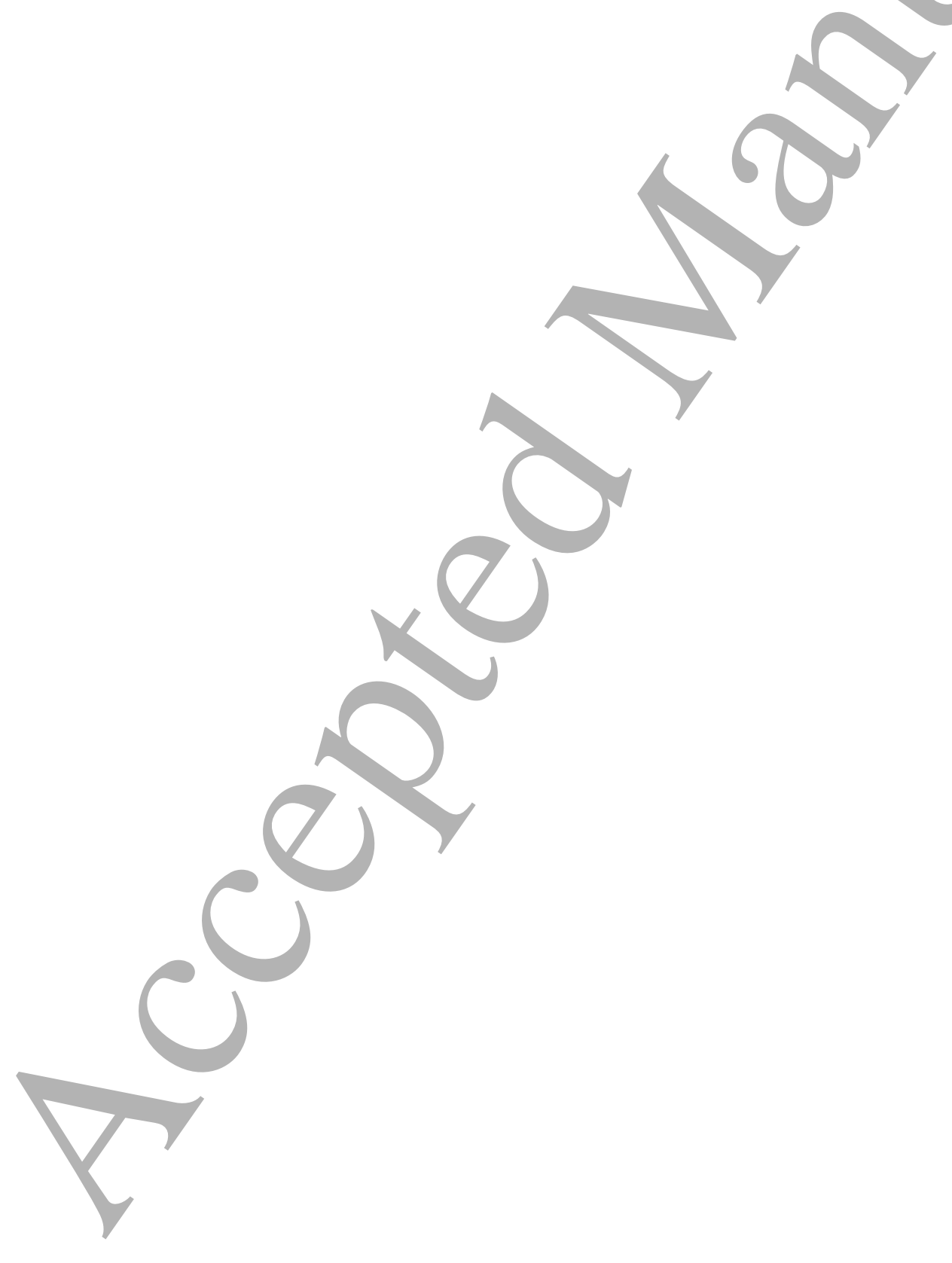




\section{Methods}

We use LBIBCell to simulate the epithelial tissue dynamics [33]. LBIBCell is an open-source C++ simulation environment (https://bitbucket.org/tanakas/lbibcell/src/master) that enables the detailed representation of 2D biological tissues at cellular resolution. As mitosis and cytokinesis are confined to the most apical epithelial region [30], our simulations focus on the apical cell dynamics (Figure 1a). We have previously described the application of LBIBCell to the simulation of the apical cell dynamics in epithelial tissues [39]; in the following, we provide a brief summary;

\section{Setup of LBIBCell simulations}

In LBIBCell, every biological process is represented by means of BioSolver (BS). We used the existing BS for cell growth, cell division, apoptosis, cortical (membrane) tension, and cell-cell adhesion [33, 39], and developed a new BS to include external forces as described below. Growth of the cells is simulated by adding mass to intracellular grid points. Cells that exceed a certain cell area are divided. The cell division threshold follows a Gaussian distribution with mean $\mu_{\text {div }}$ and standard deviation $\sigma_{\text {div }}$. The cell division plane was set either to follow a measured distribution (see below), or followed Hertwig's rule $[9,10]$, i.e. cells were divided perpendicular to their longest axis (Figure

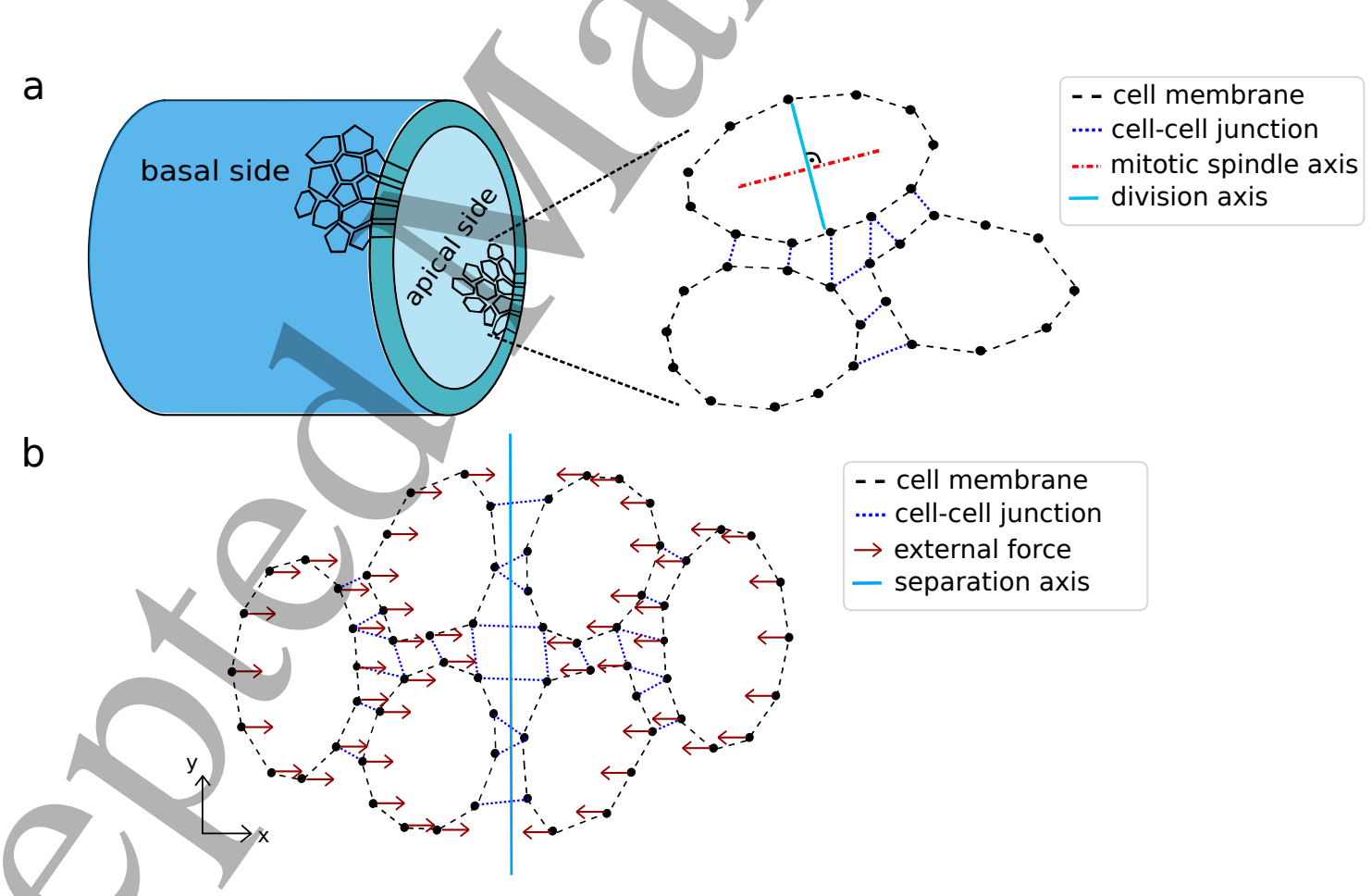

Figure 1. Schematic representations of simulated cells in LBIBCell. (a) An epithelial tube consisting of a monolayer with an outer basal side and an inner apical side facing the lumen. Cells in $\angle B I B C$ I $I$ represent the apical side of a tube. Cell cortical (membrane) tension and cell-cell junctions are represented by Hookean springs. The cell division angle is assumed to be perpendicular to the mitotic spindle axis, here the longest axis of a cell. (b) Illustration of the application of external forces. The simulated domain is split into two halves (blue line) and the forces are applied at each cell node acting in opposing directions. 
1 1a). Cells that fall below a certain cell area or that come too close to the boundaries of the simulated domain are removed. Cortical tension (called membrane tension in LBIBCell) and cell-cell adhesion are represented by Hookean spring forces between the cell membrane points (Figure 1a).

The BS involve a total of 14 free parameters (Table 1). Seven of these parameters affect numerical aspects, and these were set for maximal computational efficiency while avoiding numerical artefacts, as described before [39]. In particular, the mass added to intracellular grid points per time step is severely limited by numerical stability. We used a growth source $g=10^{-4}$ and adjusted the overall simulation time to match the data. The lung tubes expand 3.6-fold between somite stage 38 to 47 , which corresponds to about $25 \mathrm{~h}=90000 \mathrm{~s}$ [41], corresponding to a growth rate of $k \simeq 0.0515 \frac{1}{\mathrm{~h}}$, assuming exponential growth [4]. With a growth source $g=10^{-4}$ we require 36000 iteration steps to obtain a similar domain growth in the simulations, such that $2.5 \mathrm{~s} \equiv 1 \mathrm{LB}_{\text {time }}$. The cell division threshold was set such that the average cell area is $350 \mathrm{LB}_{\text {length }}^{2}$ in the simulations. With a measured average cell area of $30 \mathrm{\mu m}^{2}$ [4], the length conversion between LB units and SI units is then $0.293 \mu \mathrm{m} \equiv 1 \mathrm{LB}_{\text {length }}$. For numerical reasons, we set the minimal cell size, below which cells were removed (threshold for cell apoptosis and removal) to $20 \mathrm{LB}_{\text {length }^{2}}$. For the remaining seven parameters that describe biological processes we have previously defined parameter ranges that result in epithelia-like lattices [39], and we further set parameter values to recapitulate measurements in the mouse embryonic lung. The cell-cell junction length was set to $0.1 \mathrm{LB}_{\text {length }}=30 \mathrm{~nm}$, consistent with the measured E-cadherin length [42]. The only parameters, for which we do not have data from the embryonic mouse lung, are the spring constants. Accordingly, we studied their impact in parameter screens within a previously determined parameter range that results in epithelia-like tissues. If not stated otherwise, we set $k_{\mathrm{cj}}=0.01 \mathrm{LB}_{\frac{\text { force }}{\text { length }}}$ and $k_{\mathrm{mt}}=0.01 \mathrm{LB}_{\frac{\text { force }}{\text { length }}}$.

\section{Application of external forces}

To simulate the impact of external forces on tissue outgrowth, we implemented a new BS in LBIBCell that applies external forces to the cells, the External Forces BS. In addition to the membrane tension and the cell-cell junctions, a free force can be applied to the cell nodes, whose strength and direction are defined by a two dimensional vector. The application of this force can be coupled to cell properties such as cell type or the position of a cell. With the new BS, we split the simulated domain in two halves and apply forces in opposing directions to imitate the effect of a surrounding tissue that prevents the epithelial tube to expand in circumferential direction (Figure 1b).

\section{Cell division according to a predetermined distribution}

To study the impact of biased cell division orientation on tissue growth, we developed a Cell Division BS that applies cell division according to a predetermined distribution. In previous versions of LBIBCell, dividing cells split along an individual cell division plane whose direction is either chosen based on a random number generator or perpendicular to the longest axis. In the Cell Division BS, the mitotic spindle angle of a cell is drawn from a given mitotic spindle angle distribution. The cell division angle is perpendicular to the mitotic spindle angle. 


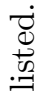

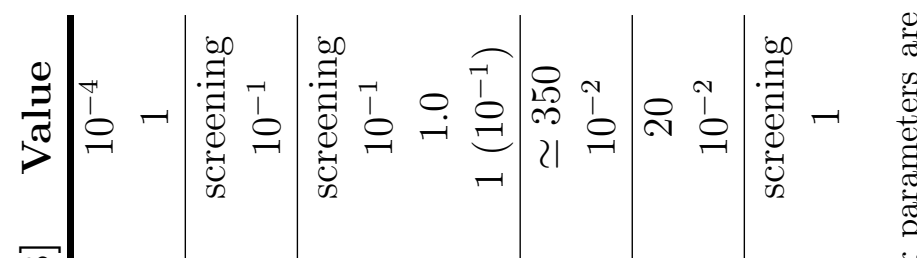

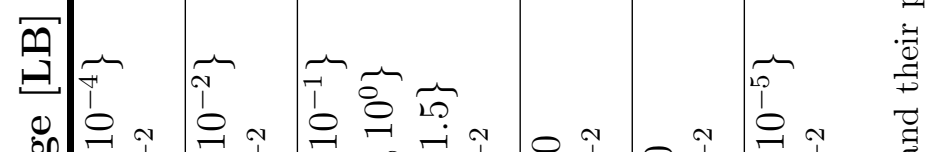

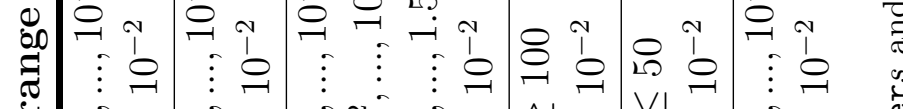

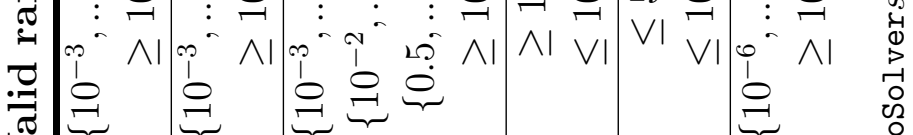

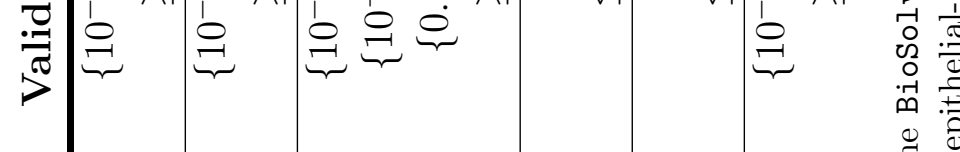

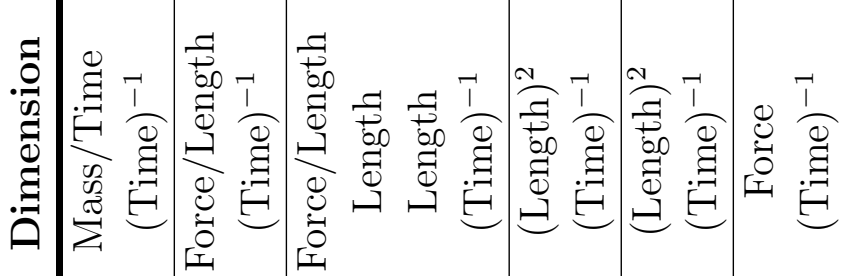

焉学

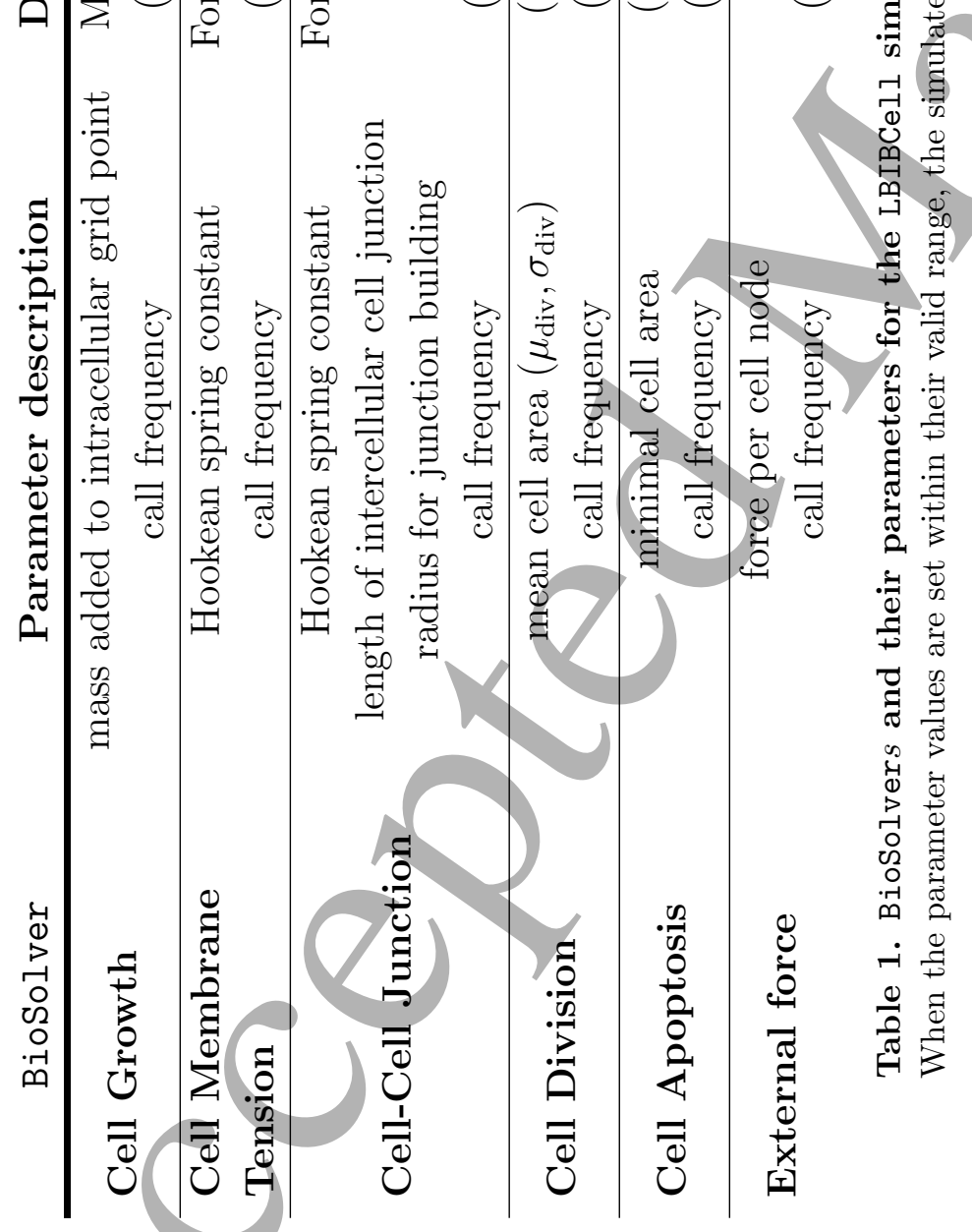


1

2

\section{Results}

\subsection{A bias in mitotic spindle orientation results in biased outgrowth only for strong cortical tension}

We sought to analyze whether the observed bias in cell shape and cell division orientation (Figure 2a-c) would be sufficient to explain the measured bias in embryonic lung tube outgrowth [4, 5]. To this end, we simulated tissue growth using parameter values that are consistent with measurements in mouse lung epithelia (Table 1), and we divided cells either according to the biased mitotic spindle angle distribution that has been experimentally measured in wildtype mouse lungs (Figure $2 \mathrm{~b}$ ) or according to the uniform mitotic spindle angle distribution of $S h h^{\text {cre } /+}$; $K r a s^{L S L /+}$ mutant (Figure 2c) mouse lung epithelia [4]. In both cases, the simulation started with an initial tissue structure which consisted of about 100 cells, had approximately the form of a square and was positioned in the middle of the simulated domain. in the case of the mitotic angle distribution from the wildtype, the final tissue adopted a rectangular tissue shape with longer sides in the respectively biased division direction (Figure 2d). On the other hand, for the experimentally measured mitotic spindle angle distribution found in $S h h^{\text {cre/+}}$; Kras ${ }^{L S L /+}$ mutant lung epithelia [4], the tissue grew over time, but preserved its square shape (Figure 2e). This demonstrates that the biased mitotic spindle angle orientation in the wildtype can result in biased tissue outgrowth.

We next sought to investigate the parameter dependency of biased outgrowth. Following [4], we define the bias in outgrowth as $\rho_{F}=\frac{\rho_{t_{2}}}{\rho_{t_{1}}}$, with $\rho_{t}=\frac{x(t)}{y(t)} ; y(t)$ denotes the length of the tissue along the elongation axis, and $x(t)$ the tissue length in the orthogonal direction. Accordingly, the bias in outgrowth along the elongation axis (y axis) is larger, the smaller $\rho_{F}$. There are only seven parameters with a biological interpretation, and these determine the growth rate, the cell area division threshold, the critical cell area below which cells delaminate or undergo apoptosis, the length of intercellular junctions, as well as the spring constants for cell-cell adhesion and cortical tension. When changing any of the non-mechanical parameters by $\pm 20 \%$, the relative changes in $\rho_{F}$ is less than $12 \%$ (Figure $3 \mathrm{~b})$. In particular, the length of intercellular cell-cell junctions and the critical cell area under which cells delaminate or undergo apoptosis have only a very small impact on the bias of outgrowth, i.e. the change in $\rho_{F}$ is less than $5 \%$. 


\section{a}

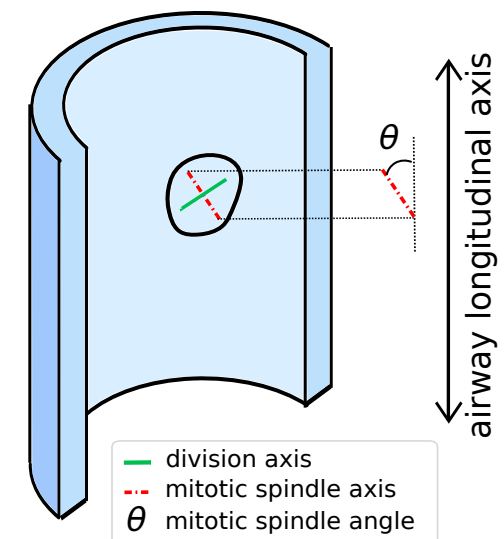

b

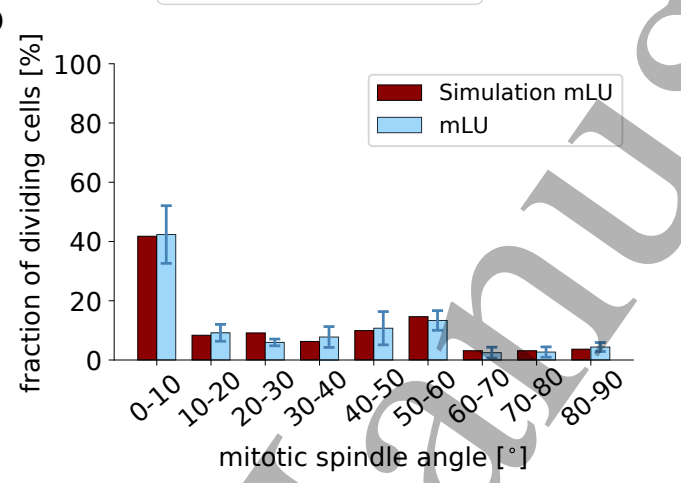

C

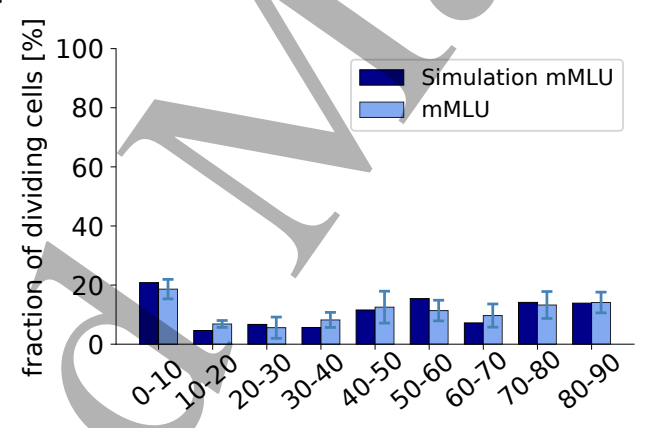

mitotic spindle angle $\left[{ }^{\circ}\right]$

d

e

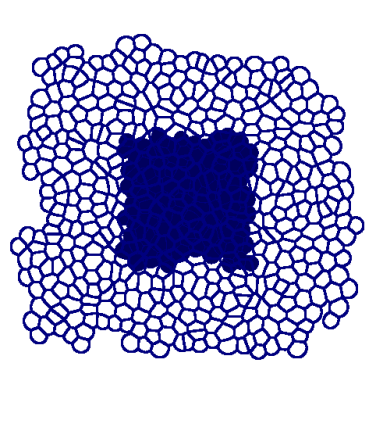

Figure 2. Oriented mitotic spindle angle distribution leads to biased tissue outgrowth. (a) Schematic representation of the mitotic spindle orientation, $\theta$, relative to the longitudinal axis of a lung tube. (b,c) Experimentally measured distribution of mitotic spindle orientations, $\theta$, from (b) wildtype mouse lungs (mLU) and (c) mutant mouse lungs (mMLU) [4] and an exemplary simulated distribution for each case. The mean and standard deviation of are shown. (d,e) Comparison of the initial tissue configuration (filled cells) with final tissue shape of the simulation, shows (d) anisotropic tissue expansion in the direction of the mitotic spindle bias (b), and (e) isotropic tissue expansion in the case of the mutant spindle angle distribution (c). 
a

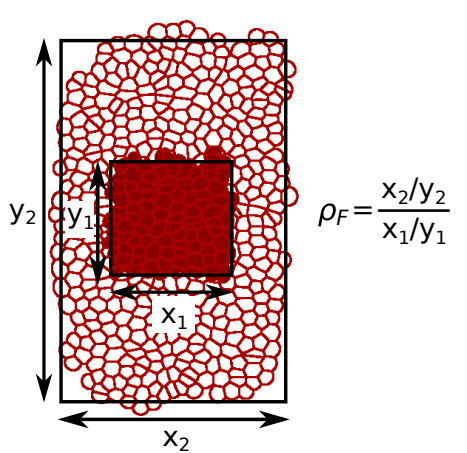

b

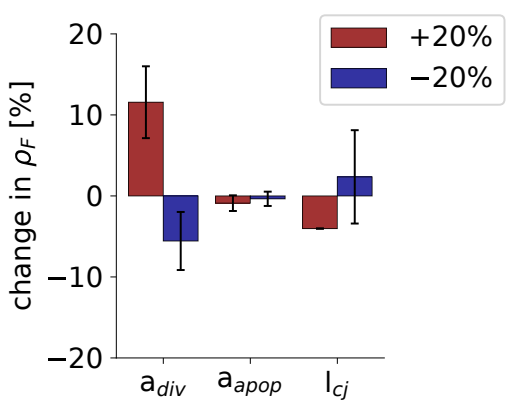

C

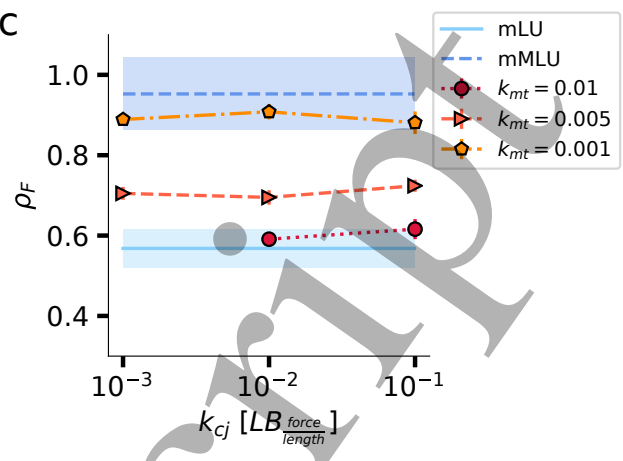

Figure 3. Parameter Sensitivity Analysis with regard to bias in tissue outgrowth. (a) To determine the bias in outgrowth, the irregularly shaped tissue is approximated by a rectangle of the same area, using a least squares fit. The bias of outgrowth is then calculated by comparing the ratio between the axes at two consecutive time points. The smaller $\rho_{F}$, the stronger is the outgrowth along the elongation axis (y axis). (b) The relative changes in the airway shape change $\rho_{F}$ for the wildtype were $<12 \%$ when changing the division area threshold, the apoptosis area and the length of cell-cell junctions by $\pm 20 \%$. The mean and the standard deviation are shown for each parameter set. (c) The airway shape change $\rho_{F}$ depends only on cortical (membrane) tension, but not on the cell-cell junction force. The simulated outgrowth ratios were compared to the measured values for wildtype (mLU) and mutant (mMLU) mouse lung epithelia [4].

We next screened the two mechanical parameters, the spring constants for cell-cell adhesion, $k_{\mathrm{cj}}$, and cortical (membrane) tension, $k_{\mathrm{mt}}$, within a range that gives rise to epithelial-like tissue (Table 1 ). We note that only a very small range of cell-cell adhesion strengths, $k_{\mathrm{cj}}$, yield an epithelial-like tissue: for lower values of the cell-cell adhesion constant, $k_{\mathrm{cj}}$, cells detach, while for higher values, cells become angularly shaped. Within this range, the cell-cell adhesion constant $k_{\mathrm{cj}}$ does not affect the bias in outgrowth (Figure 3c). On the contrary, the bias in outgrowth $\rho_{F}$ is highly sensitive to the cortical tension, $k_{\mathrm{mt}}$, and the outgrowth bias that is observed in lung epithelial tubes $\left(\rho_{F} \sim 0.6\right.$, Figure $3 \mathrm{c}$, shaded in light blue) is obtained only for the highest possible cortical (membrane) tension tension constant $k_{\mathrm{mt}}=0.01$.

Oriented divisions lead to biased outgrowth only for high cortical tension because of the importance of cell rounding. Upon cell division, the daughter cells are first elliptically shaped with the axis of cell division marking the long axis (Figure 4a). At high cortical tension, cells seek to round up. Cell rounding drives a redistribution of cell mass in the direction of the mitotic spindle axis, thus resulting in biased outgrowth (Figure 4a). For increasing cell size this effect is weaker (Figure 3b and Figure 4b). The effect is visible also in a tissue context (Figure 4c,d). At high cortical tension, the cells round up, and the rounding results in mass being directed towards the axis of elongation (Figure 4c). For low cortical tension, this cell rounding does not happen (Figure 4d), such that the tissue expands isotropically even though cells divide with a bias in the direction of elongation. Importantly, in all cases cells remain clonal, i.e. daughter cells remain in a close neighborhood.

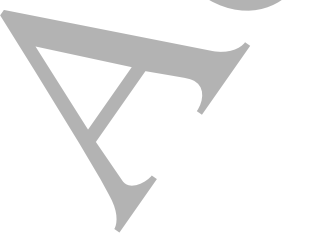




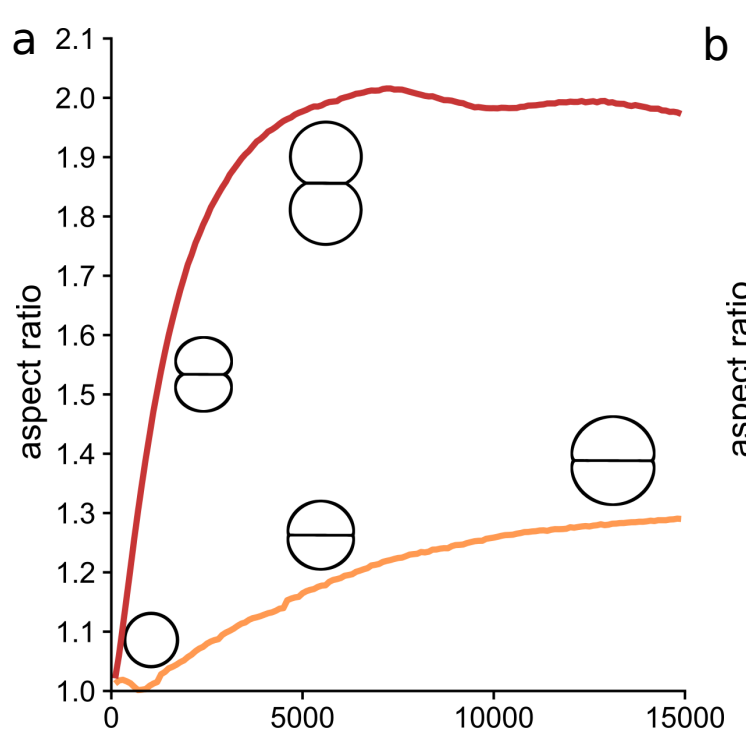

C

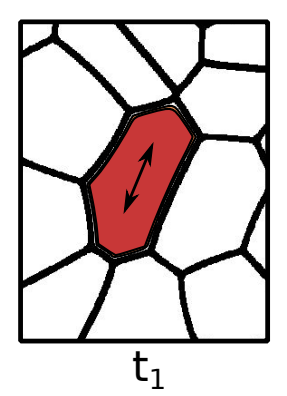
$\mathrm{LB}_{\text {time }}$

d
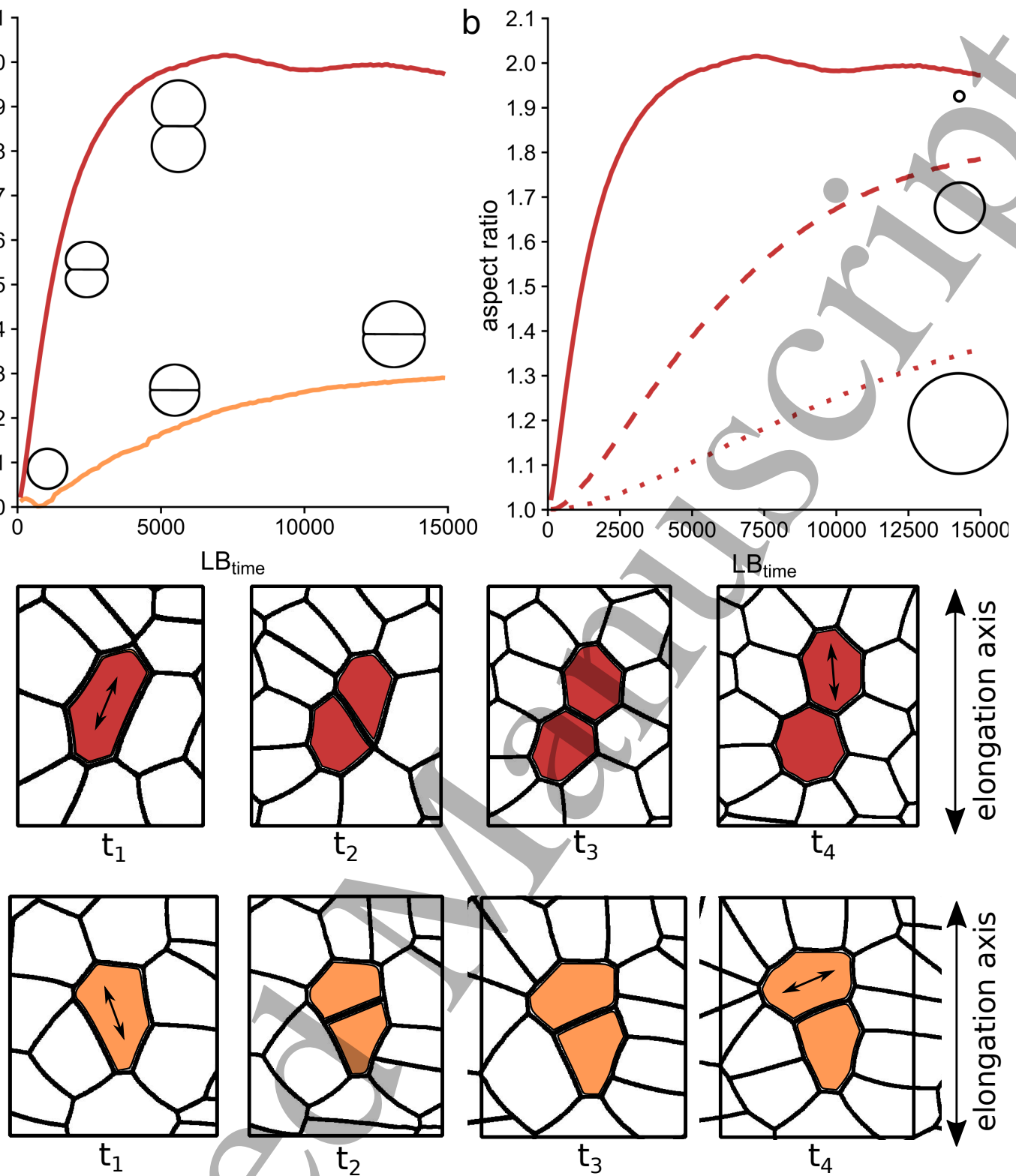

d
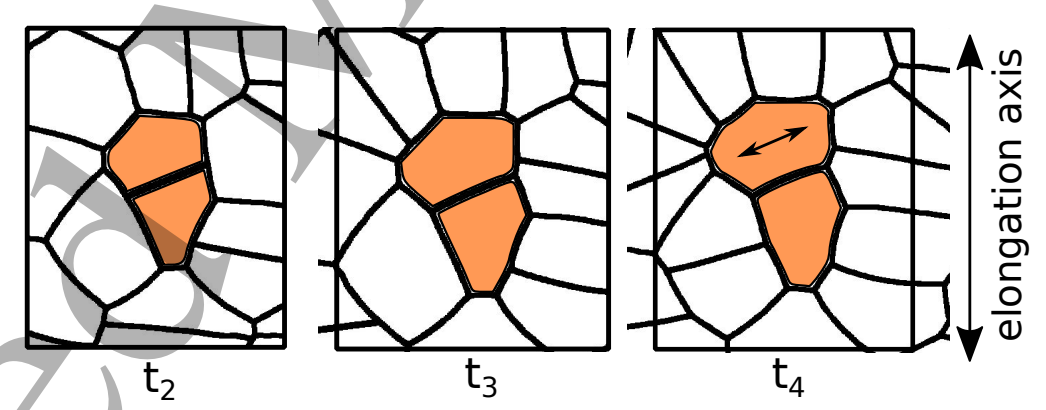

Figure 4. High cortical tension drives biased outgrowth by cell rounding after biased cell division. (a,b) High cortical (membrane) tension $k_{\mathrm{mt}}=0.01$ (a, red) causes cell rounding after cell division, which results in a redistribution of mass in direction of cell division. This effect is weaker for lower cortical tension $k_{\mathrm{mt}}=0.001$ (a, orange) and for increasing cell size (b): initial cell diameter $21 \mathrm{LB}_{\text {length }}$ (solid), $100 \mathrm{LB}_{\text {length }}$ (broken), $200 \mathrm{LB}_{\text {length }}$ (dotted). The aspect ratio was determined by fitting ellipses to the outline of both cells and calculating the ratio of the main axes. The mean and standard deviation is shown for each parameter set. (c) In a tissue with high cortical (membrane) tension, the daughter cells round up, such that mass is distributed in direction of cell division. (d) In the tissue with lower membrane tension the daughter cells retain their shape after cell division and thus expand more isotropically. 

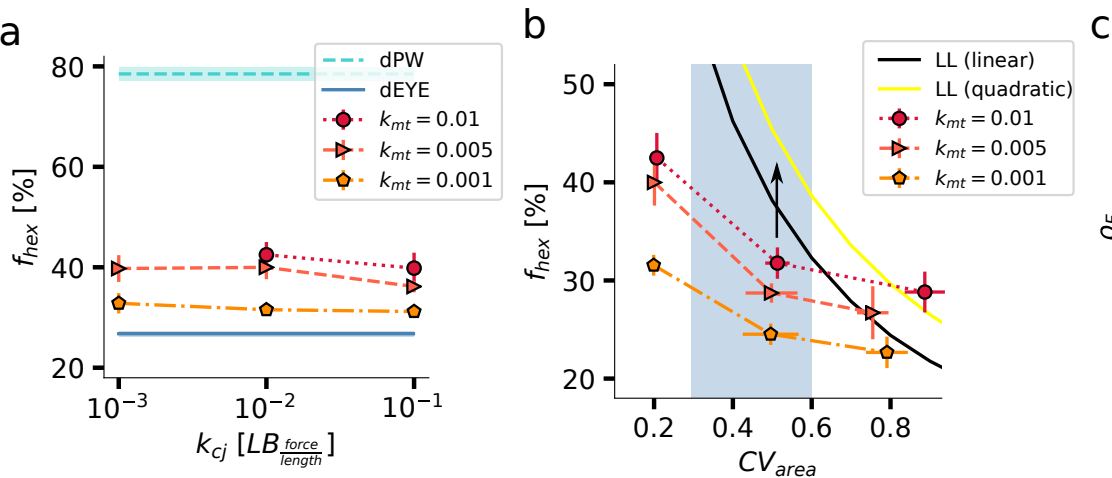

Figure 5. Comparison of simulated lattice organisation to measured epithelial organisation. (a) The hexagon frequency $f_{\text {hex }}$ depends on cortical (membrane) tension, but not on the cell-cell junction force. The hexagon frequencies in the simulations lie within the range of measured hexagon frequencies [39], i.e. Drosophila pupal wing disc (dPW) [37] and the Drosophila eye disc (dEYE) [38]. (b,c) The hexagon frequency $f_{\text {hex }}(\mathrm{b})$, and the airway shape change $\rho_{F}$ (c) are affected by the area variability: for increasing $C V_{\text {area }}$ the hexagon frequency is reduced and the bias in outgrowth is weaker. The simulated outgrowth ratios were compared to the measured values for wildtype (mLU) and mutant (mMLU) mouse lung epithelia [4]. The shaded area in (b) marks the range of observed $C V_{\text {area }}$ values [39], i.e. Drosophila pupal wing disc (dPW) with $C V_{\text {area }}=0.28$ [37], and Drosophila wing disc with gigas RNAi clones (dMWL) with $C V_{\text {area }}=0.58$. The black and yellow lines represent the analytically derived relations between the fraction of hexagons and the area variability for the linear Lewis' law (black line) and the alternative quadratic relationship (yellow line) that apply when cortical tension dominates over cell-cell adhesion.

We next wondered whether the physiological cortical tension would be high enough to support elongating outgrowth. An indirect measure of the strength of the cortical tension is the fraction of hexagons in a tissue [39]. For the high cortical (membrane) tension constant that is required for biased outgrowth, the fraction of hexagons predicted by our simulations, $f_{\text {hex }}$ is around $45 \%$. Such a fraction is indeed often observed in epithelial tissues [43], and well within the measured physiological range (Figure 5a); data for the embryonie mouse epithelium is not available.

We have previously shown that the fraction of hexagons depends not only on the membrane tension constant $k_{\mathrm{mt}}$, but also on the variability of the apical cell areas [39]. Thus, most epithelial tissues follow Lewis' law (black and yellow curves in Figure 5b), and the hexagon fraction decreases as the area variability increases. The variability of the apical areas arises mainly from cell growth, cell division, and interkinetic nuclear migration. For a constant cell division threshold in our LBIBCell simulations, the area variability is much lower (coefficient of variation $C V_{\text {area }}=\frac{\sigma_{\text {area }}}{\mu_{\text {area }}} \sim 0.2$ ) than what is observed in most tissues $\left(C V_{\text {area }}=\frac{\sigma_{\text {area }}}{\mu_{\text {area }}} \in[0.3,0.6]\right)$; here, $\mu_{\text {area }}$ refers to the mean area and $\sigma_{\text {area }}$ to its standard deviation. The area distribution can be widened by drawing the cell division threshold from a distribution rather than using a fixed value. Such widening of the area distribution results in the predicted decline in the hexagon fraction, but most simulated tissues then lie below the predicted curves (Figure 5b), which apply when tissues seek to minimize the overall cell perimeter and thus when cortical tension dominates over cell-cell adhesion. Given problems with numerical leakage, sufficiently high ratios between cortical tension and cell-cell adhesion strength can only be 
achieved in LBIBCell at high area CV (Figure 5b). The area CV affects the bias in outgrowth for the high membrane (cortical) tensions that are required to obtain the measured bias in outgrowth (Figure 5c). Due to the numerical limitations, the bias in outgrowth can only be reproduced for the lowest measured area $\mathrm{CV} \sim 0.3$. However, we can extrapolate that higher cortical tensions will allow us to reproduce the observed bias in outgrowth also for higher area CV values (Figure $5 \mathrm{~b}, \mathrm{c}$, arrows). Such higher cortical tensions likely apply in vivo as all measured epithelia/follow the predicted relationships between hexagon fraction are area CV that applies when cortical tension dominates over cell-cell adhesion (Figure 5b, black and yellow lines). We conclude that the observed bias in cell division is sufficient to explain the bias in outgrowth observed in the lung epithelium if cortical tension dominates over cell-cell adhesion.

\subsection{External compressive forces can drive bias in outgrowth, but do not give rise to the measured} bias in cell shape and cell division

We next investigated whether a constricting force from the surrounding tissue or ECM could explain the bias in cell shape, cell division orientation, and tube outgrowth in the lung epithelium. In our simulations, we applied compressive external forces to the growing cells and divided cells according to Hertwig's longest axis division rule, i.e. perpendicular to their longest axis [9]. Under these conditions, we observed a bias in tissue outgrowth (Figure 6a). For comparison, we performed simulations under the same conditions without applying external forces, and we observed isotropic tissue outgrowth (Figure 6b). The velocity field along the elongation axis (y) remains linear whether or not a compressive force is applied (Figure 6c-e), thus showing that growth remains uniform. In the presence of a compressive force $\left(F=4 \times 10^{-6} \mathrm{LB}_{\text {force }}\right)$, the velocity gradient is, however, steeper in the direction of outgrowth relative to the orthogonal direction, which is consistent with the observed biased outgrowth of $\rho_{F} \sim 0.6$ (Figure $6 \mathrm{~d}$ ).

We next analyzed to what extent cortical tension had an impact on tissue outgrowth and the cell division bias when applying compressive external forces. Here, we kept the cell-cell junction strength at $k_{\mathrm{cj}}=0.01 \mathrm{LB}_{\frac{\text { force }}{\text { length }}}$, and varied only the membrane tension constant $k_{\mathrm{mt}}$ and the external forces $F$. We found that the strength of the external force determines the bias in tissue elongation, independent of the (cortical) membrane tension (Figure 7a): the higher the external force, the stronger the outgrowth perpendicular to the direction of the force, independent of the tissue's cortical tension. The cortical tension only affected the fraction of hexagons (Figure $7 \mathrm{~b}$ ), which was slightly higher than when using the measured bias in mitotic spindle angles (Figure 5a), but still lies within the physiological range (Figure $7 \mathrm{~b}$ ). The application of external forces also affected the mitotic spindle angle distribution of a growing tissue: the fraction of biased cell divisions, i.e. the fraction of cells with a mitotic spindle angle $0^{\circ}$ to $10^{\circ}$ relative to the direction of outgrowth, increases with the applied external force (Figure 7c). However, the simulated values of cells with a biased division orientation were still much lower than the measured value in the wildtype lung and rather in the range of the measured value of the mutant lungs [4]. Plotting the bias in outgrowth, $\rho_{F}$, against the fraction of biased cell divisions shows that when the measured bias in outgrowth could be reproduced, the bias in the mitotic angles was then much lower than what was observed in the lung (Figure 7d). 
a

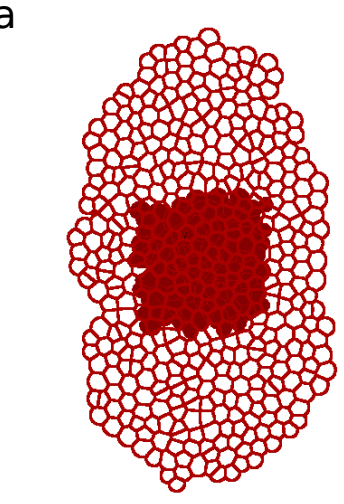

b

C
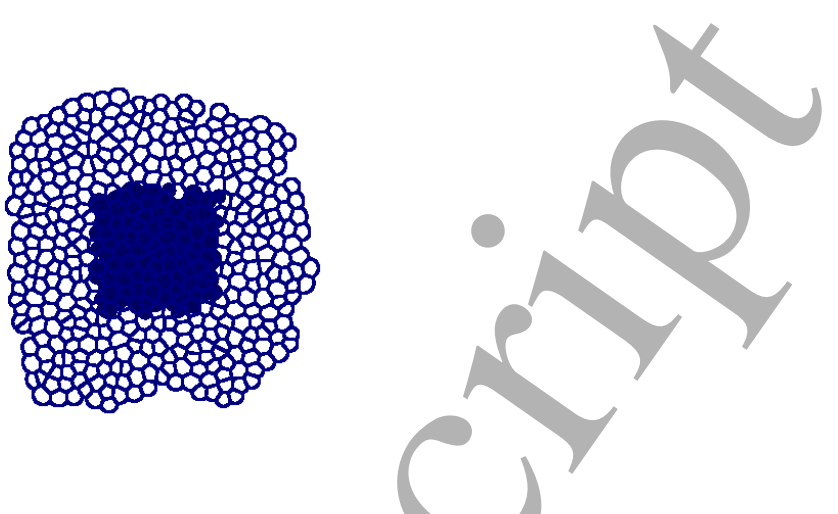

(
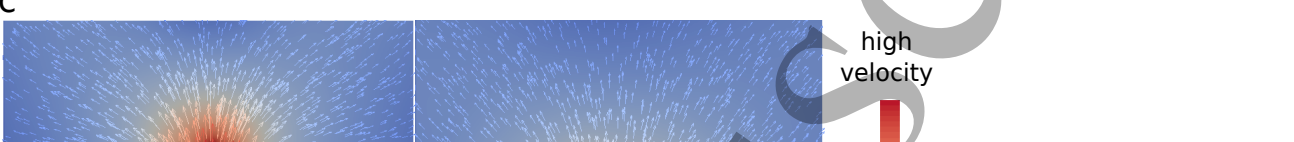

\section{d}

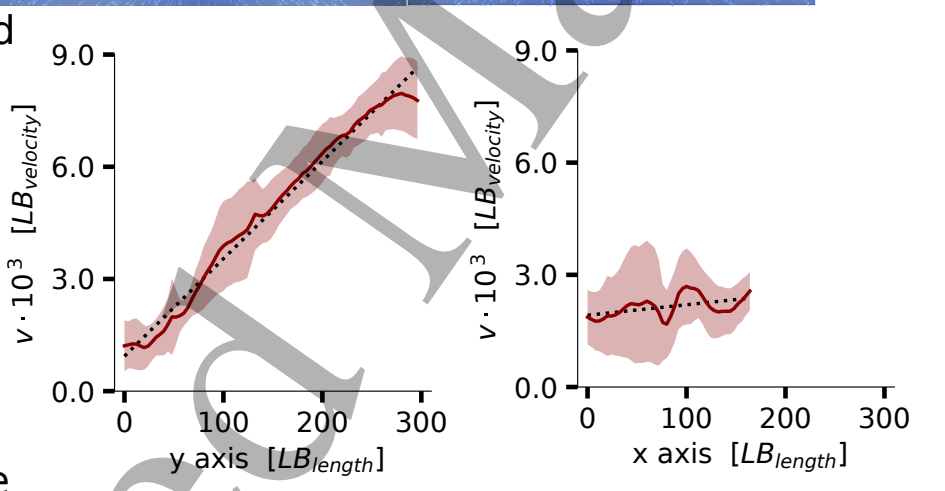

e
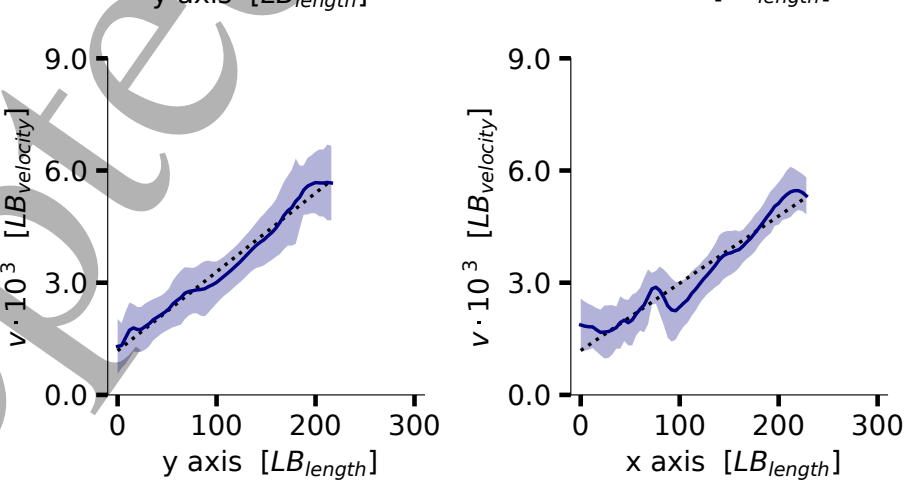

Figure 6. External compressive forces impact tissue outgrowth. (a,b) Comparison of the initial tissue configuration (filled cells) with final tissue shape of the simulations with (a) and without (b) external compressive forces. The application of external forces shows anisotropic tissue expansion in the direction perpendicular to the direction of the applied forces. Simulated tissue growth without external compressive forces results in isotropic tissue expansion. (c) Comparison of the fluid velocity field at the end of simulations with and without external compressive forces. (d) Applying external compressive forces results in a large approximately linear velocity gradient along the elongation axis (y axis) and a small approximately linear velocity gradient along the $x$ axis. (e) Without applying external compressive forces, a smaller approximately linear velocity gradient is observed. The gradient is similar along the $x$ and $y$ axis. 
1

2

3

4

5

6

7

8

9 a

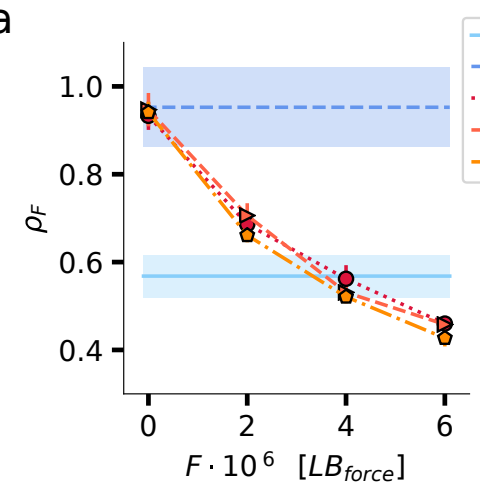

C

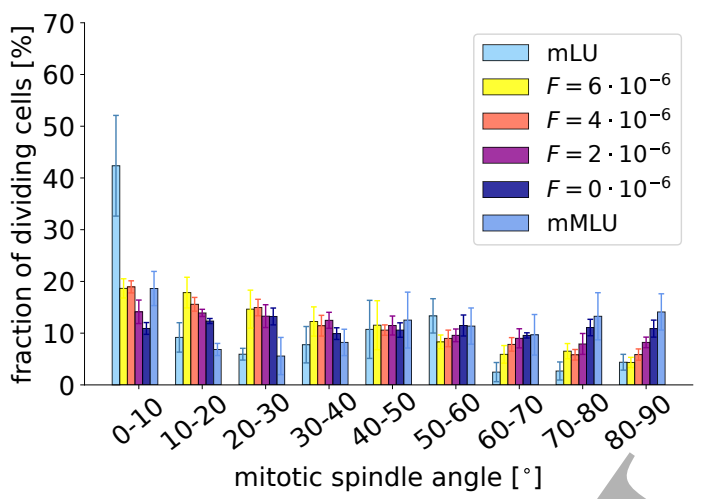

b

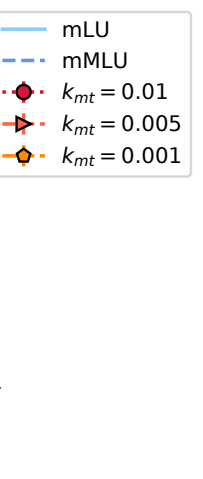

d

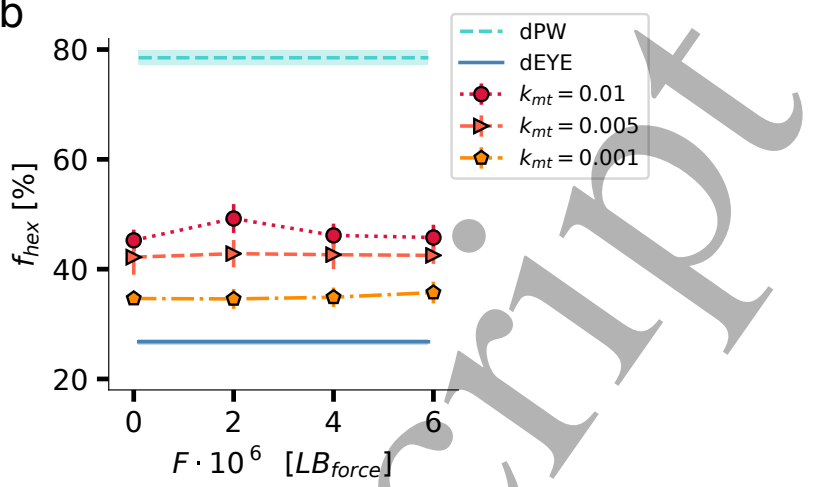

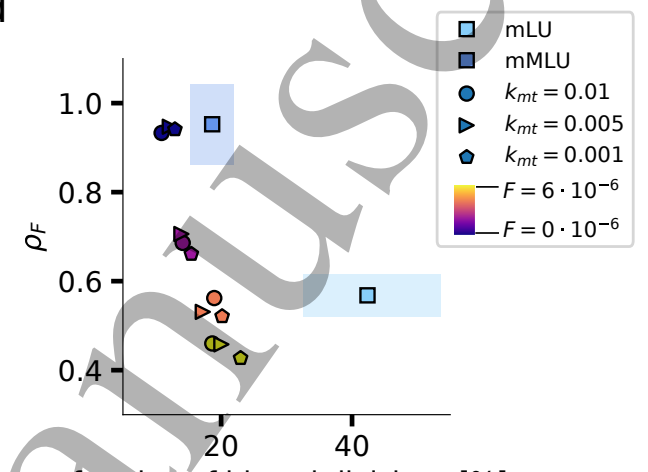

fraction of biased divisions [\%]

Figure 7. Cortical tension and external forces impact the bias of tissue outgrowth and cell division angles. (a-b) Airway shape change $\rho_{F}$ is decreasing for increasing external compressive force, while the hexagon frequency $f_{\text {hex }}$ is not affected. (c) Mitotic spindle angle distributions for high $k_{\mathrm{mt}}$ show that external compressive forces increase the fraction of biased divisions. (d) Airway shape change $\rho_{F}$ against fraction of biased divisions shows that for no combination of external force and membrane tension the measured values for wildtype (mLU) and mutant (mMLU) mouse lung epithelia [4] can be reproduced. The simulated outgrowth ratios and mitotic spindle distributions were compared to the measured values for wildtype (mLU) and mutant (mMLU) mouse lung epithelia [4] and hexagon frequencies were compared to measured values for epithelial tissues from Drosophila (pupal wing disc (dPW) [37], eye disc (dEYE) [38]).

In summary, the application of external compressive forces that restrain tissue expansion in one dimension led to biased tissue outgrowth and a bias in cell division orientation. However, there was no external force in our simulations, for which the measured bias in outgrowth and the measured bias in cell division for wildtype mouse lungs could both be captured.

Lastly, we tested the impact of the cell area variability for different membrane tensions in combination with constant external forces $\left(F=4 \times 10^{-6} \mathrm{LB}_{\text {force }}\right)$. We found that for a higher cell area variability, there is a slightly smaller bias in tissue outgrowth (Figure 8a,b). A comparison to the simulations with an intrinsic bias in the mitotic spindle angle distribution (Figure 5b) showed that the impact of cell area variability on the polygon distribution of cells within a tissue was not affected by the application of external forces (Figure 8c). Corresponding to the slightly smaller bias in tissue outgrowth, the bias in the mitotic spindle angle distribution was also slightly lower for higher cell area variabilities (Figure 8d). A higher external force is thus required for larger apical 


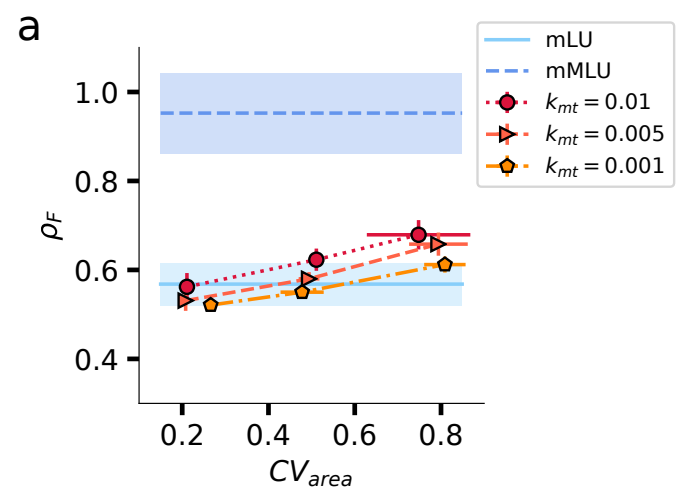

C

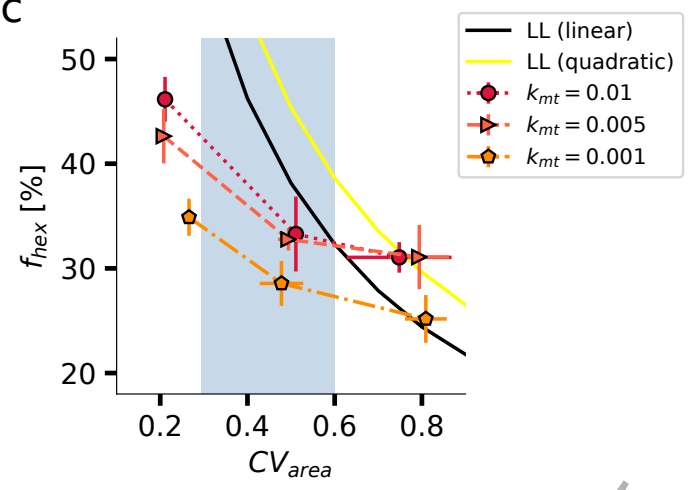

b

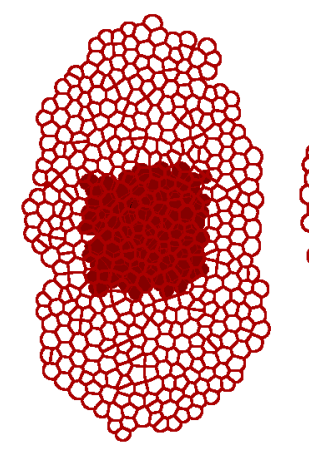

d

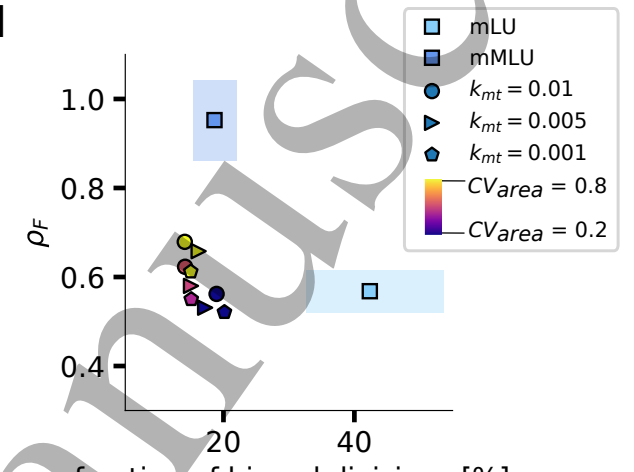

fraction of biased divisions [\%]

Figure 8. Area variability has a minor impact on the bias of tissue outgrowth and cell division angles in the presence of external forces. (a) Airway shape change $\rho_{F}$ increases for increasing area $C V$ values with $F=4 \times 10^{-6} \mathrm{LB}_{\text {force }}$. (b) Comparing the initial tissue configuration (filled cells) with final tissue shape of the simulations, shows that for higher area $C V$ values the anisotropic expansion is slightly lower. (c) Hexagon frequency $f_{\text {hex }}$ decreases for increasing $C V_{\text {area }}$. (d) Airway shape change $\rho_{F}$ against fraction of biased cell divisions b compared to the measured values for wildtype (mLU) and mutant (mMLU) mouse lung epithelia [4]. The simulated outgrowth ratios were compared to the measured values for wildtype (mLU) and mutant (mMLU) mouse lung epithelia [4] and hexagon frequencies were compared to analytically derived relations (linear and quadratic Lewis' law [39]) shown as black curve and yellow curve, respectively.

1 area variabilities to achieve the same bias in tissue outgrowth. Overall, the external force, and to 2 a small extent the area variability, determine biased tissue outgrowth and the mitotic spindle angle 3 distribution, while cortical tension and area variability mainly determine the hexagon frequency of 4 a tissue.

$5 \quad$ We conclude that biased tissue outgrowth can be achieved for any cortical tension when applying 6 compressive external forces. However, the measured bias in embryonic lung outgrowth is then 7 achieved with a much lower bias in the cell division orientation than what is observed in experiments. 8 This suggests that other processes generate the bias in the cell division orientation in the developing 9 lung, and thus drive the biased outgrowth of the epithelial lung tubes during mouse development. 


\section{Discussion}

We have used our cell-based tissue simulation framework LBIBCell to explore how biased epithelial outgrowth is achieved during mouse lung development. Experimental studies have previously linked the observed bias in outgrowth to a bias in cell shapes and cell division $[4,5]$, and the simulations demonstrate that this is indeed plausible as long as the cortical tension is sufficiently high. The requirement for high cortical tension is consistent with the observation that biased outgrowth is lost in mutants that express a constitutive active form of KRas $\left(S_{h h^{c r e /+}} ; K_{R a S^{L S L /+}}\right)$ [4]. Constitutive active KRas has previously been shown to result in a redistribution of phosphorylated myosin light chain 2 (pMLC2) inside cells of pancreatic ducts [29], which may result in reduced cortical tension on the apical side of the epithelium, where all cell divisions occur [30].

How the bias in cell shapes arises has remained unclear. We explored a possible role of constricting forces as has been proposed for the mammary gland [19] and plants [22]. While constricting forces can recapitulate the observed biased outgrowth, the resulting bias in cell shapes is much lower than what is reported for the embryonic lung epithelium. This suggests that other mechanisms drive elongating lung outgrowth. This conclusion is consistent with observations from embryonic kidneys, which show elongating outgrowth also when cultured without the surrounding mesenchyme [21]. Stretching of developing lungs in the direction of outgrowth further increases the bias in outgrowth as well as the bias in the cell division orientation [5]. Unfortunately, due to numerical limitations that arise from the low viscosity of the simulated fluid, the effects of stretching could not be studied using LBIBCell.

The biased elongation of the mammary gland has been linked to a constricting force from the myoepithelium. Based on our simulations, we would predict a low bias in cell shape in the mammary gland if this is indeed the responsible driving force. Interestingly, in the mouse embryonic neural tube, strong biased outgrowth is observed without a strong bias in cell shape [44]. The mechanism that results in biased outgrowth of the neural tube in dorsal-ventral direction is unknown [45], but our observations would support a constricting force orthogonal to the direction of biased outgrowth. As the bias in outgrowth is even higher than in the lung while the reported area $\mathrm{CV}=0.73$ is rather high in the mouse embryonic neural tube [46], a rather strong constricting force must be expected.

Going forward, it will be important to employ a combination of organ culture, live microscopy, mechanical measurements, and cell-based tissue simulations in 3D to define the mechanism that directs biased outgrowth of mammalian epithelial tubes during morphogenesis.

\section{Acknowledgments}

This work has been supported through an SNF Sinergia grant (CRSII5_170930) to D.I. The authors thank members of the Iber group for discussions.

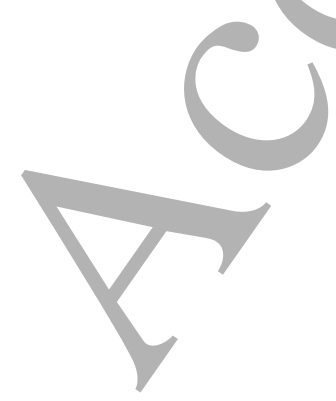




\section{References}

[1] Andrew DJ, Ewald AJ. Morphogenesis of epithelial tubes: Insights into tube formation, elongation, and elaboration. Dev Biol. 2010 may;341(1):34-55.

[2] Weavers H, Skaer H. Tip cells: Master regulators of tubulogenesis? Seminars in Cell and Developmental Biology. 2014;31:91-99.

[3] Kishimoto K, Tamura M, Nishita M, Minami Y, Yamaoka A, Abe T, et al. Synchronized mesenchymal cell polarization and differentiation shape the formation of the murine trachea and esophagus. Nat Commun. 2018;9(1):2816.

[4] Tang N, Marshall WF, McMahon M, Metzger RJ, Martin GR. Control of Mitotic Spindle Angle by the RASRegulated ERK1/2 Pathway Determines Lung Tube Shape. Science. 2011;333(July):342-345.

[5] Tang Z, Hu Y, Wang Z, Jiang K, Zhan C, Marshall WF, et al. Mechanical Forces Program the Orientation of Cell Division during Airway Tube Morphogenesis. Developmental Cell. 2018;44(3):313-325.e5.

[6] Saburi S, Hester I, Fischer E, Pontoglio M, Eremina V, Gessler M, et al. Loss of Fat4 disrupts PCP signaling and oriented cell division and leads to cystic kidney disease. Nat Genet. 2008;40(8):1010-5.

[7] Gong Y, Mo C, Fraser SE. Planar cell polarity signalling controls cell division orientation during zebrafish gastrulation. Nature. 2004;430(7000):689-93.

[8] Ciruna B, Jenny A, Lee D, Mlodzik M, Schier AF. Planar cell polarity signalling couples cell division and morphogenesis during neurulation. Nature. 2006;439(7073):220-4.

[9] Hertwig O. Das Problem der Befruchtung und der Isotropie des Eies. Eine Theorie der Vererbung. Jenaische Zeitschrift fur Naturwissenschaft. 1884;18:276-318.

[10] Minc N, Burgess D, Chang F. Influence of cell geometry on division-plane positioning. Cell. 2011;144(3):414-26.

[11] Bosveld F, Markova O, Guirao B, Martin C, Wang ZM, Pierre A, et al. Epithelial tricellular junctions act as interphase cell shape sensors to orient mitosis [Journal Article]. Nature. 2016;530(7591):495-+.

[12] Lecuit T, Lenne PF. Cell surface mechanics and the control of cell shape, tissue patterns and morphogenesis. Nat Rev Mol Cell Biol. 2007 aug;8(8):633-644.

[13] Bellusci S, Grindley J, Emoto H, Itoh/N, Hogan BL. Fibroblast growth factor 10 (FGF10) and branching morphogenesis in the embryonic mouse lung. Development. 1997 dec;124(23):4867-4878.

[14] Volckaert T, Campbell A, Dill E, Li C, Minoo P, De Langhe S. Localized Fgf10 expression is not required for lung branching morphogenesis but prevents differentiation of epithelial progenitors. Development. 2013 sep;140(18):3731-3742.

[15] Menshykau D, Kraemer C, Iber D. Branch móde selection during early lung development. PLoS Comput Biol. 2012;8(2):e1002377.

[16] Menshykau D, Blanc P, Unal E, Sapin V, Iber D. An interplay of geometry and signaling enables robust lung branching morphogenesis. Development. 2014;141(23):4526-4536.

[17] Huebner RJ, Neumann NM, Ewald AJ. Mammary epithelial tubes elongate through MAPK-dependent coordination of cell migration. Development. 2016;143(6):983-93.

[18] Ewald AJ, Brenot A, Duong M, Chan BS, Werb Z. Collective epithelial migration and cell rearrangements drive mammary branching morphogenesis. Dev Cell. 2008;14(4):570-81.

[19] Paine I, Chauviere A, Landua J, Sreekumar A, Cristini V, Rosen J, et al. A Geometrically-Constrained Mathematical Model of Mammary Gland Ductal Elongation Reveals Novel Cellular Dynamics within the Terminal End Bud. PLoS Comput Biol. 2016;12(4):e1004839.

[20] Kumar ME, Bogard PE, Espinoza FH, Menke DB, Kingsley DM, Krasnow MA. Defining a mesenchymal progenitor niche at single-cell resolution. Science. 2014;346(6211).

[21] Qiao J, Sakurai H, Nigam SK. Branching morphogenesis independent of mesenchymal-epithelial contact in the developing kidney. Proc Natl Acad Sci U S A. 1999;96(13):7330-5.

[22] Mirabet V, Das P, Boudaoud A, Hamant O. The Role of Mechanical Forces in Plant Morphogenesis. Annual Review of Plant Biology. 2011;62(1):365-385.

[23] Weijer CJ. Collective cell migration in development. Journal of Cell Science. 2009;122(18):3215-3223.

49 [24] Xiong F, Ma W, Hiscock TW, Mosaliganti KR, Tentner AR, Brakke KA, et al. Interplay of cell shape and 
1

2

3

4

5

6

7

8

9 division orientation promotes robust morphogenesis of developing epithelia. Cell. 2014;159(2):415-427.

[25] Forgacs G, Foty RA, Shafrir Y, Steinberg MS. Viscoelastic properties of living embryonic tissues: a quantitative study. Biophysical journal. 1998;74(5):2227-34.

[26] Guillot C, Lecuitial T. Mechanics of Epithelial Tissue Homeostasis and Morphogenesis. Science. 2013;p. 1185-9.

[27] Richard Brewer J, Mazot P, Soriano P. Genetic insights into the mechanisms of Fgf signaling. Genes and Development. 2016;30(7):751-771.

[28] Okudela K, Yazawa T, Suzuki T, Sugimura H, Kitamura H. Role of 3'-phosphoinositides in oncogenic KRASinduced modulation of shape and motility of airway epithelial cells. Pathology International. 2009;59(1):28-37.

[29] Messal HA, Alt S, Ferreira RMM, Gribben C, Wang VM, Cotoi CG, et al. Tissue curvature and apicobasal mechanical tension imbalance instruct cancer morphogenesis. Nature. 2019;566(7742):126-130.

[30] Ragkousi K, Gibson MC. Cell division and the maintenance of epithelial order. Journal of Cell Biology. 2014;207(2):181-188.

[31] Multerer MD, Wittwer LD, Stopka A, Barac D, Lang C, Iber D. Simulation of Morphogen and Tissue Dynamics. Methods in Molecular Biology. 2018;1863:223-250.

[32] Tanaka S. Simulation Frameworks for Morphogenetic Problems. Computation. 2015;3(2):197-221.

[33] Tanaka S, Sichau D, Iber D. LBIBCell: a cell-based simulation environment for morphogenetic problems. Bioinformatics. 2015;(March):1-8.

[34] Fletcher AG, Osterfield M, Baker RE, Shvartsman SY. Vertex models of epithelial morphogenesis [Journal Article]. Biophys J. 2014;106(11):2291-304.

[35] Chen S, Doolen GD. Lattice Boltzmann method for fluid flows. Annu Rev Fluid Mech;(Kadanoff 1986):329-364.

[36] Peskin CS. The immersed boundary method. Acta numerica. 2002;11(January 2002):479-517.

[37] Classen AK, Anderson KI, Marois E, Eaton S. Hexagonal packing of Drosophila wing epithelial cells by the planar cell polarity pathway. Developmental Cell. 2005;9(6):805-817.

[38] Heller D, Hoppe A, Restrepo S, Gatti L, Tournier AL, Tapon N, et al. EpiTools: An Open-Source Image Analysis Toolkit for Quantifying Epithelial Growth Dynamics. Developmental Cell. 2016;36(1):103-116.

[39] Kokic M, Iannini A, Fombuena GV, Casares F, Iber D. Minimisation of surface energy drives apical epithelial organisation and gives rise to Lewis' law. bioarXiv: 101101/590729;.

[40] Vetter R, Kokic M, Gomez H, Hodel L, Gjeta B, Iannini A, et al. Aboave-Weaire's law in epithelia results from an angle constraint in contiguous polygonal lattices. bioarXiv: 101101/591461;

[41] Tam PP. The control of somitogenesis in mouse embryos. J Embryol Exp Morphol. 1981;65 Suppl:103-28.

[42] Leckband DE, de Rooij J. Cadherin adhesion and mechanotransduction. Annual review of cell and developmental biology. 2014;30:291-315.

[43] Gibson MC, Patel AB, Nagpal R, Perrimon N. The emergence of geometric order in proliferating metazoan epithelia. Nature. 2006;442(7106):1038-41.

[44] Kicheva A, Bollenbach T, Ribeiro A, Valle HP, Lovell-Badge R, Episkopou V, et al. Coordination of progenitor specification and growth in mouse and chick spinal cord. Science. 2014;345(6204).

[45] Guerrero P, Perez-Carrasco R, Zagorski M, Page D, Kicheva A, Briscoe J, et al. Neuronal differentiation affects tissue mechanics and progenitor arrangement in the vertebrate neuroepithelium. bioRxiv. 2019;.

[46] Grego-Bessa J, Hildebrand J, Anderson KV. Morphogenesis of the mouse neural plate depends on distinct roles of cofilin 1 in apical and basal epithelial domains. Development. 2015;142(7):1305-1314. 